\title{
A Review of AGB Mass Loss Imaging Techniques
}

\author{
M. Marengo ${ }^{\mathrm{A}}$ \\ A Harvard-Smithsonian Center for Astrophysics, Cambridge, MA 02138, USA. \\ Email: mmarengo@cfa.harvard.edu
}

Received 2009 January 5, accepted 2009 February 7

\begin{abstract}
Circumstellar imaging across the electromagnetic spectrum allows us to derive fundamental diagnostics for the physics of mass loss in the Asymptotic Giant Branch (AGB) phase. I review the current status of the field, with particular emphasis on the techniques that provide the strongest constraints for mass-loss modeling efforts.
\end{abstract}

Keywords: circumstellar matter — stars: AGB and post-AGB — stars: mass loss

\section{Introduction}

Asymptotic Giant Branch (AGB) stars are the ancient alchemists dream: complex nucleosynthesis and mixing processes in their nuclear furnaces are the source of a variety of heavy nuclei that are ultimately released into the interstellar medium by strong stellar winds. With a cumulated rate of $\sim 0.55 \mathrm{M}_{\odot} \mathrm{yr}^{-1}$ (75\% of the total mass loss in the Galaxy), AGB stars are among the principal culprits in the heavy elements pollution of the Galaxy (Sedlmayr 1994).

Despite their importance for stellar evolution and the chemical evolution of the interstellar medium, the processes responsible for the AGB winds are not fully understood. While there is a clear link between mass loss and stellar pulsations (AGB stars tend to be Long Period Variables), the details of this relation are not completely known. The basic mechanism appears to be radiation pressure on dust grains forming in their extended atmospheres (Salpeter 1974), but a self consistent theory predicting AGB mass loss rates is still lacking. While models of dust driven winds in $\mathrm{C}$-rich $(\mathrm{C} / \mathrm{O}>1)$ AGB atmospheres are now capable of passing observational tests (see e.g. Gautschy-Loidl et al. 2004; Nowotny et al. 2005), the same does not hold true for silicate dust in an O-rich environment (Woitke 2006; Höfner \& Anderson 2007; Höfner 2008). A comprehensive set of prescriptions for mass loss rates, dust production and yields as a function of stellar parameters (including metallicity) and time are still missing and highly desirable (van Loon 2008).

Even the basic mass loss parameters (the mass loss rate, the total mass released by the star during the AGB phase, and the dust to gas mass ratio) are poorly determined by current observational techniques (Ramstedt et al. 2008; van Loon 2000). While spectral observations are crucial to determine the kinematic and composition of the AGB wind, direct imaging of the circumstellar envelopes provide unique spatial information. Images at different wavelengths allow probing the spatial structure, location and temporal variation of the mass loss 'engine', and a 'fossil record' of the AGB mass loss.

While this is not aimed to be a comprehensive review, within the next sections I will summarize recent results for current imaging techniques designed to probe the different aspects of the AGB outflows. Radio and sub-mm maps are sensitive to the molecular gas components, and are essential to estimate the total mass loss rate of the stars and its detailed chemistry (Section 2). Optical scattered light and polarimetry are instead an important tool to map the dust content of the circumstellar envelope at large distances from the star, thus providing a historical record of the mass loss rate over tens of thousand of years (Section 3). The infrared domain is sensitive to the thermal emission of the circumstellar dust. As such it is the preferred domain to probe the inner regions of the envelopes, where dust formation and growth processes are active (Section 4). High energy phenomena like jets and shocks can arise when the AGB star is part of a close binary system. Studying these structures is important to understand the physics of mass transfer and the origin of asymmetries in the AGB wind (Section 5). Section 6 instead focuses on the imaging of the star itself, in its role as engine of the mass loss processes. In Section 7 I will conclude by exploring the opportunities that will be offered for AGB circumstellar envelopes imaging with the instrumentation available within the next decade.

\section{The Molecular Envelopes}

Most of the gas phase in AGB circumstellar envelopes is in molecular form, and can be mapped with radio and millimeter-wave observations. Since the first detection of $\mathrm{OH}$ masers around evolved stars (Wilson \& Barrett 1968), and CO in the envelope of the prototype carbon star IRC+10216 (Solomon et al. 1971), a large number of molecules have been detected (Olofsson 2006). Mapping the distribution of these molecules with single dish and 
interferometric observations allows to study the geometry of the gas component in the AGB molecular outflows.

Thermally excited molecular transitions (traditionally $\mathrm{CO}$ and $\mathrm{HCN}$, but more recently also rotational lines from $\mathrm{SiO})$ are observed in AGB stars of all chemical types, and are used as reliable indicators of mass loss (Loup et al. 1993; González-Delgado 2003) and to study the general symmetry of the molecular envelope (Neri et al. 1998; Olofsson et al. 1996, 2000; Lindqvist et al. 1999; Fong et al. 2006). High angular resolution imaging of those molecules has recently provided the measurement of velocity gradients, anisotropies and chemical fractionation in the molecular envelopes (see e.g. Muller et al. 2008; Dinh-V-Trung \& Lim 2008; Schöier et al. 2006a), as well as opened a new avenue to study non-LTE chemistry and grain adsorption processes (Schöier, Olofsson \& Lundgren 2006b). Multi-epoch imaging of $\mathrm{SiO}, \mathrm{OH}, \mathrm{H}_{2} \mathrm{O}$ and other masers (see e.g. the 'movie' of $\mathrm{SiO}$ maser emission detected around the Mira variable TX Cam by Diamond \& Kemball 2003), allows to probe with sub-mas resolution the local gas infall, outflow and non-radial motion superposed to the dominant expansion of the circumstellar envelope. The polarization of $\mathrm{SiO}$ and $\mathrm{H}_{2} \mathrm{O}$ maser lines has also provided indications of stellar and circumstellar magnetic fields (see e.g. Kemball \& Diamond 1997; Vlemmings, Diamond \& van Langevelde 2001, 2002), and allowed to observe the emergence of collimated jets at the end of the AGB phase (Vlemmings, Diamond \& Imai 2006).

Observations of isotopologues of several molecules (for example ${ }^{12} \mathrm{CO}$ and ${ }^{13} \mathrm{CO}$, Milam et al. 2009 and references therein) allow to study the effects of stellar nucleosynthesis and transport processes (Soria-Ruiz et al. 2005). These observations will become very important when the Atacama Large Millimeter Array (ALMA) will be operational starting 2010. The high resolution $(\sim 0.02$ arcsec) and sensitivity of ALMA, between 0.3 and $9.6 \mathrm{~mm}$, will allow to spatially resolve the crucial isotopic and chemical gradients within the AGB circumstellar shells, reflecting the chronology of otherwise inaccessible stellar nuclear processes.

The molecular envelope of AGB and post-AGB stars can also be imaged in $\mathrm{H}_{2}$ line emission with narrow band filters at wavelengths ranging from the UV to the IR. The long tail of Mira ( $o$ Cet), tracing 30000 years of mass loss history and interactions with the interstellar medium, has been detected in far-UV $\mathrm{H}_{2}$ line with GALEX (Martin et al. 2007). Spectral $\mathrm{H}_{2}$ imaging in the infrared has been applied mostly to map the morphology of post-AGB stars and the AGB remnant halo of Planetary Nebulæ (Hrivnak et al. 2008, 2006; Sahai \& Trauger 1998; Kastner et al. 2001; Cox et al. 2003; Davis et al. 2005; Van de Steene et al. 2008). The novel technique of Integral Field Spectroscopy has been applied for these evolved objects to obtain higher spatial and spectral resolution in molecular hydrogen and other lines (Tsamis et al. 2008; Sandin et al. 2008; Santander-García et al. 2007, 2008; Sharp et al. 2006; Lowe \& Gledhill 2006; Monreal-Ibero et al. 2005).
While the gas in the AGB circumstellar envelope is mostly molecular, recent observations have been able to detect atomic lines. The 21-cm Hi neutral atomic hydrogen line, in particular, allows to probe AGB outflow at large distance from the star (Gérard \& Le Bertre 2006; Le Bertre \& Gérard 2004; Gardan et al. 2006; Matthews \& Reid 2007). Notable is the case of the detection of the HI counterpart of the $o$ Cet tail (Matthews et al. 2008). Reid \& Menten (2007) have imaged at VLA the radio continuum emission from $\mathrm{H}^{-}$free-free interactions in the stellar photosphere of 3 AGB stars, finding asymmetries in two of them (R Leo and W Hya, while $o$ Cet appeared to be spherically symmetric). These observations, if they can be obtained as time series for a larger sample of stars, will play an important role in understanding the physics at the interface between the stellar atmosphere and the circumstellar envelope, to better characterize the role of stellar pulsations in triggering the mass loss.

\section{Fossil Dust Shells in Scattered Light}

Circumstellar envelopes keep the historical record of the mass loss rate variations during the AGB phase. Far from the star the released material is not directly excited by the stellar radiation, but its dust component can still be observed at optical wavelengths in scattered light. Mauron \& Huggins $(1999,2000)$ first detected concentric shell structures around IRC +10216 , hinting to alternating phases of high and low mass loss in the last $\sim 8000 \mathrm{yr}$ with a cadence of $\sim 200-800 \mathrm{yr}$. Large aperture ground based telescopes like the VLT are allowing to probe with this technique the geometry of the envelopes and the mass loss history for an increasing number of sources at different evolutionary stage along the AGB (Mauron \& Huggins 2006).

Imaging polarimetry in the optical and in the near-IR enhance the detection of scattered light by dust grains in faint circumstellar structures by suppressing the light from the central star. Low resolution polarimetric maps of circumstellar envelopes have been obtained for more than 15 years (Kastner \& Weintraub 1993, 1994). The availability of polarimeters on the HST telescope and at large aperture ground based telescopes equipped with Adaptive Optics (AO) have recently allowed probing the asymmetry of dusty shells at sub-arcsec angular scale for sources at different evolutionary stage (see e.g. Gledhill \& Takami 2001; Gledhill 2005; Ueta, Murakawa \& Meixner 2007; Murakawa et al. 2005, 2008).

\section{Dust Thermal Radiation}

Thermal infrared is the traditional wavelength range to study the dust component of AGB circumstellar envelopes. It is no surprise that the first astronomical observations with infrared bolometers were cool evolved stars (Low \& Johnson 1964; Neugebauer, Martz \& Leighton 1965), soon recognized to be hosting circumstellar envelopes of silicate and carbonaceous dust (Wolf \& Ney 1969). The first 'image' of an AGB circumstellar envelope in the thermal infrared was the determination of the angular diameter 
of IRC +10216 by means of a lunar occultation (Toombs et al. 1972). While lunar occultations are still used today to image dust shells around AGB stars (see e.g. Harvey \& Oldag 2007), the current state of the art in high resolution thermal infrared imaging of AGB circumstellar envelopes is direct imaging with $\mathrm{AO}$ systems and interferometric techniques.

\subsection{Direct Imaging}

Thermal infrared astronomy literally took off with the launch of the InfraRed Astronomical Satellite (IRAS) in 1983. A careful analysis of IRAS data found 15 sources associated to evolved stars that were showing extended emission at 60 and $100 \mu \mathrm{m}$ (Hacking et al. 1985). A reprocessing of the IRAS data to create higher resolution maps using Pyramid Maximum Entropy (PME) image reconstruction technique (HIRAS, Bontekoe, Koper \& Kester 1994), allowed the detection of cold detached shells around three of these stars: the carbon stars U Hya (Waters et al. 1994) and U Ant (Izumiura et al. 1997) and the Orich star R Hya (Hashimoto et al. 1998). A similar analysis with the Infrared Space Observatory (ISO) photometer (ISOPHOT) revealed a dust shell around the J-type carbon star Y CVn (Izumiura et al. 1996). The age of the shells $\left(\sim 10^{3}-10^{4} \mathrm{yr}, 10\right.$ to 100 times longer than the shells detected in scattered light around IRC+10216 and other evolved AGB stars) suggests that these phases of increased mass loss may be related to the thermal pulses in the thermally pulsing-AGB phase (Willems \& de Jong 1988; Zijlstra et al. 1992). A search for more of these detached shells is currently being made using the MIPS instrument on-board the Spitzer Space Telescope (Ueta et al. 2006; Ueta 2008) and the FIS instrument on the AKARI Infrared Astronomy Satellite (Ueta et al. 2008).

The development of array cameras capable of obtaining background limited images in the $N(\sim 10 \mu \mathrm{m})$ and $Q(\sim 20 \mu \mathrm{m})$ atmospheric windows allowed the observation of AGB circumstellar dust from the ground. The first observational campaigns focused on post-AGB stars, because of their high infrared excess and large size (Dayal et al. 1998; Meixner et al. 1999; Ueta et al. 2001; Kwok, Volk \& Hrivnak 2002). Early observations of stars still on the AGB (Busso et al. 1996; Sudol et al. 1999; Marengo et al. 1999, 2001; Jura, Chen \& Plavchan 2002a, b; Lagadec et al. 2005) could only partially resolve the circumstellar envelope. Their main limiting factor was in the stability of the instrumental Point Spread Function (PSF), required for the removal of the bright stellar source to isolate the fainter circumstellar emission. This limitation was resolved with the introduction of AO systems.

The main advantage of $\mathrm{AO}$ is that it guarantees a very stable PSF by compensating for the seeing and for distortions in the optical system. In the thermal infrared this yields diffraction limited images with a very high Strehl ratio $(98 \%$ or better) which do not significantly change while moving from one target to another and to a reference star. Using AO it is possible to isolate small scale structures and detect deviations from spherical symmetry with high reliability even in sources that are only marginally extended with respect to the reference PSF (Biller et al. 2005, 2006; Leão et al. 2006; Lagadec et al. 2007).

\subsection{Interferometric Techniques}

While radio interferometry has been used for many decades to acquire astronomical images, infrared interferometry is just now reaching maturity. With interferometry it is possible to record both phases and amplitudes of incident light by combining the beams from two or more apertures. With long baseline interferometry the distance between the apertures can be of hundreds of meters, allowing to reach milli-arcsec angular resolution. For nearby AGB stars this is sufficient to resolve details of the stellar disk and map with great accuracy the anisotropies and asymmetries in the dust forming region. Interferometry techniques can also be applied to single aperture telescopes by subdividing the pupil into sub-apertures, either with a physical mask (aperture masking), or by imaging seeing speckles (speckle imaging).

The first interferometric observations of dusty circumstellar envelopes in the thermal infrared became possible with the development of the Infrared Spatial Interferometer (ISI, Danchi et al. 1990; Hale et al. 2000). ISI is unique among the infrared interferometers because it combines the light from its individual apertures (originally two truck-mounted 1.65-m movable telescopes) using a heterodyne detector with ${ }^{13} \mathrm{CO}_{2}$ lasers and local oscillators. ISI operates at $11 \mu \mathrm{m}$ with baselines ranging from 4 to $70 \mathrm{~m}$ (providing a maximum angular resolution of $\sim 20$ mas), and since 1991 (Bester et al. 1991) it has been used to derive the diameters and radial profiles for a large number of AGB circumstellar envelopes. Recently, the interferometer has been equipped with a third telescope, allowing it to use closure phases as a mean to record with great accuracy the symmetry of the observed sources (Weiner et al. 2006a; Tatebe et al. 2006; Chandler et al. 2007a,b).

The MIDI instrument (Leinert et al. 2003) operates in the $N$ and $Q$ infrared bands by combining the light from two of the VLTI telescopes (either the large 8-m UTs or the 1.8-m ATs) with baselines ranging from 8 to $200 \mathrm{~m}$. This provides an angular resolution of up to $\sim 20$ mas within a field of view of up to $\sim 1$ arcsec in radius. The limiting sensitivity of the instrument is $\sim 9$ mag in the $N$ band ( $\sim 10 \mathrm{mJy}$ ). MIDI is equipped with narrow band filters, a prism and a grism, providing maximum spectral resolution of $\sim 530$. In the last few years the instrument has been used extensively to measure the distribution of circumstellar dust around AGB and post-AGB stars. Among the highlights of these observations, the distribution of silicate dust around a carbon star (Ohnaka et al. 2008) and the detection of circumbinary disks in AGB and post-AGB stars (Deroo et al. 2006, 2007a,b).

The two Keck telescopes are also capable of working as an interferometer at infrared wavelength (Colavita et al. 2003). With a baseline of $\sim 85 \mathrm{~m}$ it provides an angular resolution of $\sim 27$ mas in the $N$ band. This system has 
been recently equipped with a low resolution spectrometer $(\Delta \lambda / \lambda \simeq 35)$ providing wavelength dependent fringe visibilities across the $8-12-\mu \mathrm{m}$ spectral range (Mennesson et al. 2005). The Keck interferometer can also operate as a nulling interferometer (KNI, Serabyn et al. 2004), in which case the light coming from the two telescopes is made to interfere destructively, in order to cancel out the central point source. This technique has been tested successfully at the MMT telescope to observe the circumstellar environment of evolved stars (Hinz et al. 1998), and a dedicated instrument is being built for the Large Binocular Telescope (LBT, Hinz et al. 2008). The KNI is already accepting proposals, while the LBT interferometer is currently in construction.

The main limitation of long baseline interferometry is the small number of apertures that can be combined in the current setups. Because of this the source coverage in Fourier space is generally not sufficient to generate true images of the observed targets. The Magdalena Ridge Observatory Interferometer (MROI) in New Mexico is being built from the ground up to be an imaging interferometer (Creech-Eakman et al. 2008). The MROI will operate in the optical and near-IR, in order to access key gas and dust diagnostic emission. It will initially have six 1.4-m aperture telescopes (10 when completed) distributed in a reconfigurable array (like the VLA), to achieve angular resolution from 0.1 to 100 mas. Thanks to these multiple baselines, it will be able to efficiently generate true images (like current radio interferometers), for sources as faint as $14 \mathrm{mag}$ in the $H$ band $(1.6 \mu \mathrm{m})$. The interferometer is on track to produce 'first light' images by 2010 .

While the new generation of imaging interferometers is still in construction, diffraction limited images have been produced at infrared wavelengths with large aperture telescopes without $\mathrm{AO}$, using speckle imaging and aperture masking. The angular resolution is not as high as in the case of long baseline interferometry, but is sufficient to probe for symmetry and anisotropy in the circumstellar envelopes. The working principle of speckle interferometry is the acquisition of short time exposures that are then combined in Fourier space (Weigelt 1977; Lohman, Weigelt \& Wirnitzer 1983; Nisenson 1988). Aperture masking consists in dividing the aperture of a single mirror telescopes in sub-apertures via a physical non-redundant mask. Image reconstruction techniques are then used to recover the final high resolution images (Haniff et al. 1997; Tuthill et al. 2000).

Both techniques have been used in the near-IR to obtain images of AGB and post-AGB circumstellar dusty envelopes with an angular resolution of $\sim 50-70$ mas. Weigelt et al. (1998, 2002) and Tuthill et al. (2000, 2005), in particular, followed the evolution of inhomogeneous and clumpy mass loss processes within the dust shell of IRC +10216 , with a spatial resolution of $\sim 5 \mathrm{AU}$ (a few stellar radii). These observations provide a unique look into the time variability of dust condensation in the AGB outflow, showing extensive departures from spherical symmetry at the level of the dust formation zone.

The presence of the 'warm' mask, which is a strong source of thermal noise, limits the utility of aperture masking in the thermal IR. The technique of 'segment tilting' (Monnier et al. 2004; Weiner et al. 2006b), solves this issue by re-orienting groups of the segments of a large aperture telescope like Keck to produce interferograms from which independent visibility amplitudes and closure phases can be measured. This technique has been used to obtain 8-, 9.9-, 10.7- and 12.5- $\mu \mathrm{m}$ images of $o$ Cet, resolving the wind accretion from Mira A to its low mass companion and detecting a possible long lived disk around Mira B (Ireland et al. 2007).

\section{Jets and Shocked Gas}

In a symbiotic system mass transfer from the evolved giant to a more compact hot star typically proceeds via Bondi-Hoyle capture. Only a small fraction of the wind is accreted through this process, which can only be detected because of the UV radiation emitted by the compact companion. Occasionally, however, this picture changes as the symbiotic system goes into an outburst which can have an optical amplitude of several magnitudes. The remnants of these dramatic events, which involve bipolar outflows with speed in excess of $1000 \mathrm{~km} \mathrm{~s}^{-1}$, can be directly imaged by the HST in narrow band ionized gas filters (see e.g. Corradi et al. 2001; Sahai et al. 2003), by radio interferometers via synchrotron radiation or thermal bremsstrahlung of the shocked gas (see e.g. Crocker et al. 2001) and in soft- and hard-band X-rays (see e.g. Karovska et al. 2005, 2007; Kellogg et al. 2007).

Imaging the outbursts in nearby symbiotic systems, that can be spatially resolved with much greater details than jets in more distant sources like YSOs or QSOs, provides important observational tests for astrophysical jets models. The peculiarity of AGB mass loss processes and wind accretion in binary systems is also important for the role played by these phenomena in the creation of asymmetric post-AGB and planetary nebulae, and 'extrinsic' AGB stars polluted by the mass transfer from a companion on the AGB phase.

\section{Imaging the Star}

Given that, ultimately, the AGB mass loss engine is the AGB star itself, many of the open questions concerning the geometry of the wind and its relationship with the stellar pulsations can be investigated by imaging the stellar photosphere and the molecular layers immediately on top of the photosphere. This has been possible for quite a long time. The same interferometric techniques described in Section 4.2, at optical and near-IR wavelengths, can resolve the stellar disks with large aperture telescopes. At $700 \mathrm{~nm}$, for example, a 6-m telescope can achieve an angular resolution of $\sim 30$ mas, sufficient to resolve the disk of nearby AGB stars. Narrow band imaging through the whole electromagnetic spectrum is particularly useful to isolate individual atomic and molecular layers in 
the photospheres, measuring changes in size and asymmetry. It can also investigate surface brightness variations in the stellar photosphere (including hot and dark spots) and derive very precise measurements of stellar limb darkening and effective temperatures, which are important constraints for AGB dynamic atmosphere modeling.

Michelson interferometers have been used for a long time to measure the diameter of giant stars (including AGBs, see e.g. the Pease 1931 measurement of the $o$ Cet diameter) at optical wavelengths. This measurements are carried on to determine stellar diameter variations in the TiO optical bands with the COAST interferometer (Burns et al. 1998; Young et al. 2000). In the near-IR these measurements are done with the IOTA/FLUOR (van Belle et al. 1996, 1997; van Belle, Thompson \& Creech-Eakman 2002; Perrin et al. 1999), PTI (Thompson, Creech-Eakman \& van Belle 2002a; Thompson, Creech-Eakman \& Akeson 2002b), VLTI (Boboltz \& Wittkowski 2005; Woodruff et al. 2004; Richichi \& Wittkowski 2003) and Keck (Eisner et al. 2007) interferometers. A strong wavelength dependence in the diameter of these stars was noticed when simultaneous observations with broad and narrow band filters (Mennesson et al. 2002; Perrin et al. 2004; MillanGabet et al. 2005; Wittkowski et al. 2008; Eisner et al. 2007) became possible. With closure phases (as in the case of IOTA/FLUOR and VLTI/AMBER), the measure of asymmetry is much more precise, and even image reconstruction can be attempted (see e.g. Ragland et al. 2006, 2008).

Speckle interferometry and aperture masking have also been used in the optical to resolve the disk of AGB stars (see e.g. Karovska et al. 1991; Weigelt et al. 1996; Wilson et al. 1992; Tuthill et al. 1994; Tuthill, Haniff \& Baldwin 1995, 1999; Haniff, Scholz \& Tuthill 1995; Bedding et al. 1997 and references therein). As mentioned in Section 2, AGB $\mathrm{H}^{-}$free-free photospheres have also been detected in the radio by Reid \& Menten (2007), opening another venue to measure stellar asymmetries.

It is finally worth noting that interferometric techniques have also been used in space to measure the diameter and symmetry of AGB optical photospheres. The Fine Guidance Sensor (FGS) on-board the Hubble Space Telescope (HST) was used to derive the optical angular diameter of AGB stars with interferometric techniques, finding strong evidences of asymmetry for R Leo and W Hya (Lattanzi et al. 1997). For the nearest stars, in fact, the stability of the PSF of the HST is sufficient to resolve the stellar photosphere with direct optical and UV imaging (see e.g. the image of $o$ Cet obtained by Karovska et al. 1997).

\section{The Future}

From the brief review presented in these pages it is clear that meaningful studies of AGB mass loss can be greatly improved by simultaneous multi-wavelength observations. Due to their Long Period Variability, AGB stars change their radius by a significant fraction on timescales of days. Modeling data sets collected at different epochs is complicated by these changes: to match spectral observations with high resolution imaging the two datasets must be obtained at nearly the same time (see e.g. Wittkowski et al. 2007; Karovikova et al. 2008).

New imaging facilities will become available within the next decade, opening new opportunities to map AGB circumstellar envelopes from the ground and from space. ALMA and MROI will provide high sensitivity and angular resolution in the infrared and sub-mm. The BLT and the KNI will make nulling interferometry of circumstellar dust practical. A new generation of 20-30-m segmented telescopes equipped with $\mathrm{AO}$ will increase the sensitivity and resolution achievable with direct imaging. These breakthroughs in ground based astronomy will be matched by new space instrumentation. The MIRI instrument onboard the James Webb Space Telescope (JWST) will provide diffraction limited narrow band imaging between 5 to $27 \mu \mathrm{m}$, sampled on a pixel size of $\sim 0.11$ arcsec. The NIRCam/JWST camera will instead operate between 0.6 to $5 \mu \mathrm{m}$, providing Nyquist sampled images with resolution of $0.06-0.13$ arcsec. Both instruments will be equipped with cononographs, a feature that is essential to suppress the bright central AGB star and detect the faint circumstellar emission. These observations will be complemented at sub-mm wavelengths by the imaging photometers of the Herschel Space Telescope, that will have limited angular resolution but unprecedented sensitivity to detect cold detached shells.

The development of high angular resolution imaging techniques like interferometry and adaptive optics, at wavelengths ranging from the $\mathrm{X}$-rays to the radio, has opened in the last decade the possibility to study with great detail the physics of mass loss in AGB and postAGB stars. The complex view that is arising from these new observations is certainly a challenge for stellar modelers, but also an opportunity to finally understand one of the most important, but also neglected, ingredients in stellar and galactic evolution.

\section{Acknowledgments}

I would like to thanks the organizers of the workshop 'The Origin of the Elements Heavier than Iron' for the wonderful time spent in Torino, and Roberto Gallino, to whom the workshop was dedicated, and the whole stellar group in Torino for their support while I was taking my first steps in astrophysics.

\section{References}

Biller, B. A. et al., 2006, ApJ, 647, 464

Biller, B. A. et al., 2005, ApJ, 620, 450

Bedding, T. R., Zijlstra, A. A., von der Luhe, O., Robertson, J. G., Marson, R. G., Barton, J. R. \& Carter, B. S., 1997, MNRAS, 286, 957

Bester, M., Danchi, W. C., Degiacomi, C. G. \& Townes, C. H., 1991, ApJ, 367, L27

Boboltz, D. A. \& Wittkowski, M., 2005, ApJ, 618, 953

Bontekoe, Tj. R., Koper, E. \& Kester, D. J. M., 1994, A\&A, 284, 1073 
Busso, M. et al., 1996, A\&A, 311, 253

Burns, D. et al., 1998, MNRAS, 297, 462

Chandler, A. A., Tatebe, K., Wishnow, E. H., Hale, D. D. S. \& Townes, C. H., 2007a, ApJ, 670, 1347

Chandler, A. A., Tatebe, K., Hale, D. D. S. \& Townes, C. H., 2007b, ApJ, 657, 1042

Colavita, M. et al., 2003, ApJ, 592, L83

Corradi, R. L. M., Munari, U., Livio, M., Mampaso, A., Gon calves, D. R. \& Schwarz, H. E., 2001, ApJ, 560, 912

Cox, P., Huggins, P. J., Maillard, J.-P., Muthu, C., Baciller, R. \& Forveille, T., 2003, ApJ, 586, L87

Creech-Eakman, M. J. et al., 2008, SPIE, 7013, 26

Crocker, M. M., Davis, R. J., Eyres, S. P. S., Bode, M. F., Taylor, A. R., Skopal, A. \& Kenny, H. T., 2001, MNRAS, 326, 781

Danchi, W. C., Bester, M., Degiacomi, C. G., McCullough, P. R. \& Townes, C. H., 1990, ApJ, 459, L59

Davis, C. J., Smith, D. M., Gledhill, T. M. \& Varricatt, W. P., 2005, MNRAS, 360, 104

Dayal, A., Hoffmann, W. F., Bieging, J. H., Hora, J. L., Deutsch, L. K. \& Fazio, G. G., 1998, ApJ, 492, 603

Deroo, P. et al., 2006, A\&A, 450, 181

Deroo, P., Acke, B., Verhoelst, T., Dominik, C., Tatulli, E. \& van Winckel, H., 2007a, A\&A, 474, L45

Deroo, P., van Winckel, H., Verhoelst, T., Min, M., Reyniers, M. \& Waters, L. B. F. M., 2007b, A\&A, 467, 1093

Diamond, P. J. \& Kemball, A. J., 2003, ApJ, 599, 1372

Dinh-V-Trung \& Lim, J., 2008, ApJ, 678, 303

Eisner, J. A. et al., 2007, ApJ, 654, L77

Fong, D., Meixner, M., Sutton, E. C., Zalucha, Z. \& Welch, W. J., 2006, ApJ, 652, 1626

Gardan, E., Gérard, E. \& Le Bertre, T., 2006, MNRAS, 365, 245

Gautschy-Loidl, R., Höfner, S., Jørgensen, U. G. \& Hron, J., 2004, A\&A, 422, 289

Gérard, E. \& Le Bertre, T., 2006, AJ, 132, 2566

Gledhill, T. M., 2005, MNRAS, 356, 883

Gledhill, T. M. \& Takami, M., 2001, MNRAS, 328, 266

González-Delgado, D., Olofsson, H., Kerschbaum, F., Schöier, F. L., Lindqvist, M. \& Groenewegen, M. A. T., 2003, A\&A, 123, 147

Hacking et al., 1985, PASP, 97, 616

Hale, D. D. S. et al., 2000, ApJ, 537, 998

Haniff, P. G., Scholz, M. \& Tuthill, P. G., 1995, MNRAS, 276, 640

Haniff, C. A., Mackay, C. D., Titterington, D. J., Sivia, D., Baldwin, J. E. \& Warner, P. J., 1987, Natur, 328, 694

Hashimoto, O., Izumiura, H., Kester, D. J. M. \& Bontekoe, Tj. R., 1998, A\&A, 329, 213

Harvey, P. M. \& Oldag, A., 2007, ApJ, 663, 543

Hinz, P. M., Solheid, E., Oli, D. \& Hoffmann, W. F., 2008, SPIE 7013, 7013339

Hinz, P. M. et al., 1998, Natur, 395, 251

Höfner, S., 2008, A\&A, 491, L1

Höfner, S. \& Andersen, A. C., 2007, A\&A, 465, L39

Hrivnak, B. J., Smith, N., Su, K. L. \& Sahai, R., 2008, ApJ, 688, 327

Hrivnak, B. H., Kelly, D. M., Su, K. Y. L., Kwok, S. \& Sahai, R., 2006, ApJ, 650, 237

Ireland, M. J. et al., 2007, ApJ, 662, 651

Izumiura, H., Waters, L. B. F. M., de Jong, T., Loup, C., Bontekoe, Tj. R. \& Kester, D. J. M., 1997, A\&A, 323, 449

Izumiura, H., Hashimoto, O., Kawara, K., Yamamura, I. \& Waters, L. B. F. M., 1996, A\&A, 315, L221

Jura, M., Chen, C. \& Plavchan, P., 2002a, ApJ, 569, 964

Jura, M., Chen, C. \& Plavchan, P., 2002b, ApJ, 574, 963

Karovikova, I., Wittkowski, M., Boboltz, D. A. \& Scholz, M., 2008, in Proc. Cool Stars 15, in press (astro-ph/0809.4619)

Karovska, M., Carilli, C. L., Raymond, J. C. \& Mattei, J. A., 2007, ApJ, 661, 1048

Karovska, M., Schlegel, E., Hack, W., Raymond, J. C. \& Wood, B. E., 2005, ApJ, 623, L137

Karovska, M., Hack, W., Raymond, J. \& Guinan, E., 1997, ApJ, 482, L175
Karovska, M., Nisenson, P., Papaliolios, C. \& Boyle, R. P., 1991, ApJ, 374, L51

Kastner, J. H., Weintraub, D. A., Gatley, I. \& Henn, L., 2001, ApJ, 546,279

Kastner, J. H. \& Weintraub, D. A., 1993, ASPC, 45, 151

Kastner, J. H. \& Weintraub, D. A., 1994, ApJ, 434, 719

Kellogg, E. et al., 2007, ApJ, 664, 1079

Kemball, A. J. \& Diamond, P. J., 1997, ApJ, 481, L111

Kwok, S., Volk, K. \& Hrivnak, B. J., 2002, ApJ, 573, 720

Lattanzi, M. G., Munari, U., Whitelock, P. A. \& Feast, M. W., 1997, ApJ, 485, 328

Lagadec, E. Chesneau, O., Zijlstra, A., Matsuura, M. \& Mékarnia, D., 2007, in Proc. of Asymmetric Planetary Nebulæ IV, in press (astro-ph/0708.3112)

Lagadec, E., Mékarnia, D., de Freitas Pacheco, J. A. \& Dougados, C., 2005, A\&A, 433, 553

Leão, I. C., de Laverny, P., Mékarnia, D., De Medeiros, J. R. \& Vandame, B., 2006, A\&A, 455, 187

Le Bertre, T. \& Gérard, E., 2004, A\&A, 419, 549

Leinert, Ch. et al., 2003, Msngr, 112, 13

Lindqvist, M., Olofsson, H., Lucas, R., Schöier, F. L., Neri, R., Bujarrabal, V. \& Kahane, C., 1999, A\&A, 351, L1

Lohman, A. W., Weigelt, G. \& Wirnitzer, B., 1983, ApOpt, 22, 4028

Loup, C., Forveille, T., Omont, A. \& Paul, J. F., 1993, A\&ASS, 99, 291

Low, F. J. \& Johnson, H. L., 1964, ApJ, 139, 1130

Lowe, K. T. E. \& Gledhill, T. M., 2006, in Planetary Nebulæ in our Galaxy and Beyond, Proc. IAU Symposium No. 234, Eds. Barlow, M. J. \& Méndez, R. H. (Cambridge: Cambridge University Press), 451

Marengo, M., Karovska, M., Fazio, G. G., Hora, J. L., Hoffmann, W. F., Dayal, A. \& Deutsch, L. K., 2001, ApJ, 556, L47

Marengo, M., Busso, M., Silvestro, G., Persi, P. \& Lagage, P. O., 1999, A\&A, 348, 501

Martin, D. G. et al., 2007, Natur, 448, 780

Matthews, L. D. \& Reid, M. J., 2006, AJ, 133, 2291

Matthews, L. D., Libert, Y., Gérard, E., Le Bertre, T. \& Reid, M. J., 2008, ApJ, 684, 603

Mauron, N. \& Huggins, P. J., A\&A, 349, 203

Mauron, N. \& Huggins, P. J., 2000, A\&A, 359, 707

Mauron, N. \& Huggins, P. J., 2006, A\&A, 452, 257

Meixner, M. et al., 1999, ApJS, 122, 221

Mennesson, B. et al., 2002, ApJ, 579, 446

Mennesson, B. et al., 2005, ApJ, 634, L169

Milam, S. N., Woolf, N. J. \& Ziury, L. M., 2009, ApJ, 690, 837

Millan-Gabet, R. et al., 2005, ApJ, 620, 961

Monnier, J. D., Tuthill, P. G., Ireland, M. J., Cohen, R. \& Tannirkulam, A., 2004, BAAS, 205, 1715

Monreal-Ibero, A., Roth, M. M., Schönberner, D., Steffen, M. \& Böhm, P., 2005, ApJ, 628, L139

Muller, S., Dinh-V-Trung, He, J.-H. \& Lim, J., 2008, ApJ, 684, L33

Murakawa, K., Suto, H., Oya, S., Yates, J. A., Ueta, T. \& Meixner, M., 2005, A\&A, 436, 601

Murakawa, K., Nakashima, J., Ohnaka, K. \& Deguchi, S., 2007, A\&A, 470, 957

Murakawa, K., Ohnaka, K., Driebe, T., Hoffmann, K.-H., Oya, S., Schertl, D. \& Weigelt, G., 2008, A\&A, 489, 195

Neri, R., Kahane, C., Lucas, R., Bujarrabal, V. \& Loup, C., 1998, A\&ASS, 130, 1

Neugebauer, G., Martz, D. E. \& Leighton, R. B., 1965, ApJ, 142, L339

Nisenson, P., 1988, in Proc. NATO Advanced Study Institute, Eds. Alloin, D. M. \& Marriotti, J.-M. (Dordrecht:Kluwer), 157

Nowotny, W., Aringer, B., Höfner, S., Gautschy-Loidl, R. \& Windsteig, W., 2005, A\&A, 437, 273

Olofsson, H., 2006, RvMA, 19, 75

Olofsson, H., Bergman, P., Eriksson, K. \& Gustafsson, B., 1996, A\&A, 311, 587 
Olofsson, H., Bergman, P., Lucas, S., Eriksson, K., Gustafsson, B. \& Bieging, J. H., 2000, A\&A, 353, 597

Ramstedt, S., Schöier, F. L., Olofsson, H. \& Lundgren, A. A., 2008, A\&A, 645, 657

Ohnaka, S., Izumiura, H., Leinert, Ch., Driebe, T., Weigelt, G. \& Wittkowski, M., 2008, A\&A, 490, 173

Pease, F. G., 1931, ErNW, 10, 84

Perrin, G. et al., 1999, A\&A, 345, 221

Perrin, G. et al., 2004, A\&A, 426, 279

Ragland, S. et al., 2006, ApJ, 652, 650

Ragland, S. et al., 2008, ApJ, 679, 746

Reid, M. J. \& Menten, K. M., 2007, ApJ, 671, 2068

Richichi, A. \& Wittkowski, M., 2003, Ap\&SS, 286, 219

Sahai, R., Morriss, M., Knapp, G. R., Young, K. \& Barnbaum, C., 2003, Natur, 426, 261

Sahai, R. \& Trauger, J. T., 1998, AJ, 116, 1357

Salpeter, E. E., 1974, ApJ, 193, 585

Sandin, C., Schönberner, D., Roth, M. M., Steffen, M., Böhm, P. \& Monreal-Ibero, A., 2008, A\&A, 486, 545

Santander-García et al., 2007, A\&A, 465, 481

Santander-García et al., 2008, A\&A, 485, 117

Serabyn, E. et al., 2004, SPIE, 5491, 806

Sharp, R. G., Reilly, N. J., Kable, S. H. \& Schmidt, T. W., 2006, ApJ, 639, 194

Schöier, F. L., Fong, D., Olofsson, H., Zhang, Q. \& Patel, N., 2006a, ApJ, 649, 965

Schöier, F. L., Olofsson, H. \& Lundgren, A., 2006b, A\&A, 454, 247

Sedlmayr, E., 1994, LNP, 428, 163

Soria-Ruiz, R., Colomer, F., Alcolea, J., Bujarrabal, V., Desmurs, J.-F. \& Marvel, K. B., 2005, A\&A, 432, L39

Solomon, P., Jefferts, K. B., Penzias, A. A. \& Wilson, R. W., 1971, ApJ, 163, 53

Sudol, J. J., Dyck, H. M., Stencel, R. E., Klebe, D. I. \& CreechEakman, M. J., 1999, ApJ, 117, 1609

Tatebe, K., Chandler, A. A., Hale, D. D. S. \& Townes, C. H., 2006, ApJ, 652, 666

Thompson, R. R., Creech-Eakman, M. J. \& van Belle, G. T., 2002a, ApJ, 577, 447

Thompson, R. R., Creech-Eakman, M. J. \& Akeson, R. L., 2002b, ApJ, 570, 373

Toombs, R. I., Becklin, E. E., Frogel, J. A., Law, S. K., Porter, F. C. \& Westphal, J. A., 1972, ApJ, 173, L71

Tsamis, Y. G., Walsh, J. R., Péquignot, D., Barlow, M. J., Danziger, J. J. \& Liu, X.-W., 2008, MNRAS, 386, 22

Tuthill, P. G., Haniff, C. A., Baldwin, J. E. \& Feast, M. W., 1994, MNRAS, 266, 745

Tuthill, P. G., Haniff, C. A. \& Baldwin, J. E., 1995, MNRAS, 277, 1541

Tuthill, P. G., Haniff, C. A. \& Baldwin, J. E., 1999, MNRAS, 306, 353
Tuthill, P. G., Monnier, J. D., Danchi, W. C. \& Lopez, B., 2000, ApJ, 543, 284

Tuthill, P. G., Monnier, J. D. \& Danchi, W. C., 2005, ApJ, 624, 352

Ueta, T., 2008, ApJ, 687, L33

Ueta, T. et al., 2006, ApJ, 648, L39

Ueta, T. et al., 2001, ApJ, 557, 831

Ueta, T., Murakawa, K. \& Meixner, M., 2007, AJ, 133, 1345

Ueta T. et al., 2008, PASJ, 60, 407

Van de Steene, G. C., Ueta, T., van Hoof, P. A. M., Reyniers, M. \& Ginsburg, A. G., 2008, A\&A, 480, 775

van Belle, G. T., Thompson, R. R. \& Creech-Eakman, M. J., 2002, ApJ, 124, 1706

van Belle, G. T., Dyck, H. M., Benson, J. A. \& Lacasse, M. G., 1996, AJ, 112, 214

van Belle, G. T., Dyck, H. M., Thompson, R. R., Benson, J. A. \& Kannappan, S. J., 1997, AJ, 114, 2150

van Loon, J. Th., 2000, A\&A, 354, 125

van Loon, J. Th., 2008, MmSAI, 79, 412

Vlemmings, W. H. T., Diamond, P. J. \& van Langevelde, H. J., 2001, A\&A, 375, L1

Vlemmings, W. H. T., Diamond, P. J. \& van Langevelde, H. J., 2002, A\&A, 394, 2002

Vlemmings, W. H. T., Diamond, P. J. \& Imai, H., 2006, Natur, 440,58

Waters, L. B. F. M., Loup, C., Kester, D. J. M., Bontekoe, Tj. R. \& de Jong, T., 1994, A\&A, 218, L1

Weigelt, G., Balega, Y. Y., Blöcker, T., Hofmann, K.-H., Men'shchikov, A. B. \& Winters, J. M., 2002, A\&A, 392, 131

Weigelt, G., 1977, OptCo, 21, 55

Weigelt, G., Balega, Y., Hofmann, K.-H. \& Scholz, M., 1996, A\&A, 316, L21

Weigelt, G., Balega, Y., Blöcker, T., Fleischer, A. J., Osterbart, R. \& Winters, J. M., 1998, A\&A, 333, L51

Weiner, J. et al., 2006a, ApJ, 636, 1067

Weiner, J. et al., 2006b, ApJ, 636, 1037

Willems, F. J. \& de Jong, T., 1988, A\&A, 309, L39

Wilson, W. J. \& Barrett, A. H., 1968, Sci, 161, 3843

Wilson, R. W., Baldwin, J. E., Buscher, D. F. \& Warner, P. J., 1992 MNRAS, 257, 369

Wittkowski, M., Boboltz, D. A., Ohnaka, K., Driebe, T. \& Scholz, M., 2007, A\&A, 470, 191

Wittkowski, M., Boboltz, D. A., Driebe, T., Le Bouquin, J.-B., Millour, F., Ohnaka, K. \& Scholz, M., 2008, A\&A, 479, L21

Woitke, P., 2006, A\&A, 460, L9

Wolf, N. J. \& Ney, E. P., 1969, ApJ, 155, L181

Woodruff, H. C. et al., 2004, A\&A, 421, 703

Young, J. S. et al., 2000, MNRAS, 318, 381

Zijlstra, A., Loup, C., Waters, L. B. F. M. \& de Jong, T., 1992, A\&A, 265, L5 